\title{
The specificity of the immunodeficiency in lepromatous leprosy
}

\author{
L J REITAN \\ National Veterinary Institute, Oslo, Norway
}

One widely used technique for in vitro tests of cell-mediated immune responses in leprosy has been to stimulate lymphocyte cultures with specific antigens. Using an isolated cell wall fraction of Mycobacterium leprae, called MLW 1, we found that this preparation was a more potent stimulator in the lymphocyte stimulation test than sonicated or whole $M$. leprae bacilli (1). This preparation induced strong lymphocyte responses in patients with untreated tuberculoid leprosy and in healthy contacts of leprosy patients, but no response in untreated patients with lepromatous leprosy.

In this same group of lepromatous leprosy patients a relatively high number of low responders to tuberculin PPD was observed. This has been noted by many workers earlier, but has been either disregarded (2) or taken as a generalized impairment of cell-mediated immunity (3). These low PPD responses could either be due to a general depression of cellmediated immune responses, or be a result of antigenic crossreactivity between PPD and $M$. leprae. To clarify these questions four additional antigens unrelated to $M$. leprae, were included. Healthy contacts of leprosy patients served as a control group.

By giving the median response of the group of healthy contacts the value of $100 \%$, the median response in \% for each group of patients could be calculated for each antigen separately. The results showed that after long term treatment lymphocytes from patients with lepromatous leprosy still failed to respond both to whole $M$. leprae bacilli and also to MLW 1 antigen (4).

M. leprae has several antigenic components in common with other mycobacteria, and cross-reactions are therefore likely to occur. Tuberculin PPD is among the most widely used immunological reagents, and the major constituent of tuberculin PPD which precipitates in antibody reations, has been shown to be an antigen corresponding to BCG60 (5). The activity of PPD that induces cell-mediated immune reactions may well be confined to this component. This antigen cross-reacts extensively with antigen no 7 of $M$. leprae in antibody reactions (6). On the other hand in cellular immune reactions we saw in some of the leprosy patients a clear dissociation in the lymphocyte responses to MLW1 and PPD, indicating that each of these two antigens contain determinants not present in the other one (1).

A marked depression of the median response to PPD in the group of untreated lepromatous leprosy patients was observed (4). After treatment the median response was still somewhat lower than in the group of healthy contacts, but the difference was no longer significant. This suggests that responses to determinants in the PPD that are not present in MLW 1, are partly restored after treatment; whereas the failure to respond to determinants shared with $M$. leprae remains. Although there is great variation in the responses, the depression of the median PPD response was seen at all three doses of PPD included, reflecting the heterogenicity of this group. 
The responses to the non-mycobacterial antigens in the untreated lepromatous leprosy group were not significantly depressed compared with the healthy contacts with one exception, the amoeba antigen. Furthermore, the responses to the Candida and Mumps antigens were significantly enhanced in the treated group compared with the untreated group. So it seems that the cross-reacting PPD antigen behaves differently from the four non-mycobacterial antigens.

The results confirm that the defect in cell-mediated immune responses in lepromatous leprosy patients is $M$. leprae specific and permanent. Although it is specific the defect also affects the response to tuberculin PPD probably reflecting the antigenic relationship between M. leprae and $\mathrm{BCG} / M$. tuberculosis.

The mechanisms behind the depression of the PPD response may be many. However, the results by Ottenhoff et al. (7) might indicate that cross-reactive T suppressor clones reacting with $M$. leprae, could play an essential role in the cell-mediated unresponsiveness in lepromatous leprosy. In this context our findings could partly be explained by $M$. leprae induced T suppressor cells affecting the PPD responses, as well, due to cross-reacting determinants.

Our findings of depressed PPD responses in a relatively hight number of lepromatous leprosy patients called for further studies comparing the in vitro lymphocyte responses to MLW1 and tuberculin PPD in individuals with different degrees of exposure to leprosy bacilli (8).

Three groups of contacts of leprosy patients were studied:

1) Close contacts. They were all family members of patients with lepromatous leprosy and were living in the same household as the patient.

2) Healthy occupational contacts.

3) Non-close contacts of leprosy patients. They were not in regular contact with the patients. Non-exposed individuals served as a control group.

A wide variation was seen in the responses to all three doses of MLW1 in all groups of contacts. Although the close contact group gave the highest median responses at all three doses, there were no significant differences between the three groups of contacts. To all three doses the median responses in the non-exposed group were markedly and significantly lower than any of the contact groups.

Also in the responses to tuberculin PPD in the same groups of individuals, a wide variation in the responses was observed. The median response in the close contacts group were markedly lower than in any of the groups to all three doses of the antigen. Thus the pattern of response to tuberculin PPD in the close contact group has some similarities with that of the untreated lepromatous leprosy patients.

There were no depression of the responses to the non-specific mitogen PHA and the nonmycobacterial amoeba antigen in the group of close contacts compared with the other groups. The mean responses to both stimulants were in fact highest in this group, showing no evidence of a non-specific depression of the immune response.

It is important to elucidate further whether this decrease in the PPD responses is an indicator for an individual with a high risk of developing disease or an indicator of subclinical infection. In a study by Nelson et. al. (9) it has been indicated that children from households of lepromatous leprosy patients are less responsive to BCG than those from households of only tuberculoid patients or normal persons. Among the children who were initially tuberculin negative, significantly decreased PPD conversion rates occurred in children from lepromatous families compared to those from families of tuberculoid patients or normals. 
The demonstration of a decreased responsiveness to PPD in the close contact group is of the greatest interest (8). Because of the heavy exposure to $M$. leprae in that particular group of contacts, it might be that their ability to respond to those determinants of PPD which are shared with $M$. leprae is reduced, and probably to $M$. leprae specific determinants as well. One explanation might be that $\mathrm{T}$ suppressor cells affecting both the specific responses to MLW1 and the cross-reacting responses to PPD, are operating.

Crucial questions concerning the mechanism of the specific immunodeficiency in lepromatous leprosy remain to be answered. Particular emphasis should be paid to events occurring early after infection and to mechanisms of apparent predisposition for developing deficiency.

Depressed PPD responses might in this context be an indicator for $M$. leprae suppression and predisposition for developing deficiency.

\section{References}

1 Closs O, Reitan LJ, Negassi K, Harboe M, Belehu A. In vitro stimulation of lymphocytes in leprosy patients, healthy contacts of leprosy patients, and subjects not exposed to leprosy. Comparison of an antigen fraction prepared from Mycobacterium leprae and tuberculin purified protein derivative. Scand J Immunol, 1982, 16, 103-115.

2 Godal T, Myklestad B, Samuel DR, Myrvang B. Characterization of the cellular immune defect in lepromatous leprosy: A specific lack of circulating Mycobacterium leprae-reactive lymphocytes, Clin exp Immunol, 1971, 9, 821-831.

3 Han SH, Weiser RS, Lin YC. Transformation of leprous lymphocytes by leprolin, tuberculin and phytohaemagglutinin. Int J Lepr, 1971, 39, 789-795.

4 Reitan LJ, Closs O, Belehu A. In vitro lymphocyte stimulation in patients with lepromatous and borderline tuberculoid leprosy. The effect of dapsone treatment on the response to Mycobacterium leprae antigens, tuberculin purified protein derivative and non-mycobacterial stimulants. Int J Lepr, 1982, 50, 455-467.

5 Closs O, Harboe M, Axelsen NH, Bunch-Christensen K, Magnusson M. The antigens of Mycobacterium bovis, strain BCG, studied by crossed immunoelectrophoresis: a reference system. Scan J Immunol, 1980, 12, 249-263.

6 Harboe M, Closs O, Bjorvatn B, Kronvall G, Axelsen NH. Antibody response in rabbits to immunization with Mycobacterium leprae. Infect Immun, 1977, 18, 792-805.

7 Ottenhoff THM, Elferink DG, Klatser PR, De Vries RRP. Cloned suppressor T cells from a lepromatous leprosy patient suppress Mycobacterium leprae reactive helper $\mathrm{T}$ cells. Nature, 1986, 322, 462-464.

8 Reitan LJ, Closs O. In vitro stimulation of lymphocytes with an antigen fraction prepared from Mycobacterium leprae and tuberculin PPD in contacts and non-contacts of leprosy patients. Clin exp Immunol, 1984, 57, 315-323.

9 Nelson KE, Speck SM, Suprasert S, Smith T. A study of cellular immunity in clinically healthy children of parents with leprosy in Northern Thailand. Int J Lepr, 1984, 52, 147-153. 